\title{
A note on Sinnott's index formula
}

\author{
by
}

Kazuhiro Dohmae (Tokyo)

0. Introduction. Let $k$ be an imaginary abelian number field with exactly two ramified primes. The letters $E$ and $C$ denote the group of units and the group of circular units in $k$ respectively. Sinnott's index formula for this case is the following (see Proposition 4.1, Theorem 4.1 and Theorem 5.1 in $[6])$.

TheOREM A (Sinnott). Let $k$ be an imaginary abelian number field with conductor $m=p_{1}^{e_{1}} p_{2}^{e_{2}}$, where $p_{1}$ and $p_{2}$ are distinct prime numbers and both $e_{1}$ and $e_{2}$ are positive integers. Denote by $k_{i}(i=1,2)$ the maximal subfield of $k$ which is unramified outside $p_{i} \infty$. Let $G$ be the Galois group of $k$ over $\mathbb{Q}$. Further, $T_{p_{i}}$ and $D_{p_{i}}$ denote the inertia group and the decomposition group of $p_{i}$ in $G(i=1,2)$. Then the group $C$ has finite index in $E$, and

(1) $[E: C]=\frac{\left[k_{1}: \mathbb{Q}\right]\left[k_{2}: \mathbb{Q}\right]}{[k: \mathbb{Q}]} \cdot 2^{-g^{\prime}} \cdot 2^{\varepsilon_{1}\left[G: D_{p_{1}}\right]+\varepsilon_{2}\left[G: D_{p_{2}}\right]+\delta_{1}+\delta_{2}-1} \cdot Q h^{+}$,

where $Q$ is the unit index of $k, h^{+}$the class number of the maximal real subfield of $k$ and $g^{\prime}$ some rational integer. Moreover, $\varepsilon_{i}$ and $\delta_{i}$ are defined by

$$
\begin{aligned}
\varepsilon_{i} & = \begin{cases}0 & \text { if } k_{3-i} \text { is imaginary, } \\
1 & \text { otherwise, }\end{cases} \\
\delta_{i} & = \begin{cases}0 & \text { if } k_{3-i} \text { is real and }\left[D_{p_{i}}: T_{p_{i}}\right] \text { is odd, } \\
1 & \text { otherwise, }\end{cases}
\end{aligned}
$$

for $i=1,2$. Finally, the rational integer $g^{\prime}$ satisfies $\mu \leq g^{\prime} \leq \nu$, where

$$
\begin{gathered}
\mu=\sharp\left\{1 \leq i \leq 2: k_{i} \text { is imaginary }\right\}, \\
\nu=\sharp\left\{1 \leq i \leq 2:\left[k_{i}: \mathbb{Q}\right] \text { is even }\right\} .
\end{gathered}
$$

In general, the formula (1) contains the unknown factor $2^{-g^{\prime}}$. But if both $k_{1}$ and $k_{2}$ are imaginary, then we have $\mu=\nu=2$ and $g^{\prime}=2$. Hence,

1991 Mathematics Subject Classification: Primary 11R27. 
in this case, (1) reads

$$
[E: C]=\frac{\left[k_{1}: \mathbb{Q}\right]\left[k_{2}: \mathbb{Q}\right]}{[k: \mathbb{Q}]} \cdot 2^{-1} \cdot Q h^{+} .
$$

In a previous paper [1], we gave another proof of (2) by constructing a system of fundamental circular units (i.e., a basis of the free part of $C$ ) of $k$. It is the main purpose of this note to prove the following completely explicit version of Theorem A.

Theorem B. Let the notation be as in Theorem A. Then

$$
[E: C]=\frac{\left[k_{1}: \mathbb{Q}\right]\left[k_{2}: \mathbb{Q}\right]}{[k: \mathbb{Q}]} \cdot 2^{\varepsilon_{1}\left[G: D_{p_{1}}\right]+\varepsilon_{2}\left[G: D_{p_{2}}\right]-1} \cdot Q h^{+} .
$$

The proof includes the explicit construction of a system of fundamental circular units of $k$. By the way, comparing (1) with (3), we obtain

$$
g^{\prime}=\delta_{1}+\delta_{2}
$$

Kučera kindly wrote me a direct proof of the inequality $g^{\prime} \geq \delta_{1}+\delta_{2}$. But I have never found any direct proof of (4) .

In the last section, we also mention our result on a real abelian number field with exactly two ramified primes.

1. Notation. Let $k$ be an imaginary abelian number field of conductor $m=p_{1}^{e_{1}} p_{2}^{e_{2}}$. We note that $k$ is a subfield of the $m$ th cyclotomic field $K=$ $\mathbb{Q}\left(\zeta_{m}\right)$, where $\zeta_{m}=e^{2 \pi \sqrt{-1} / m}$. Let $N$ be the subgroup of $T=(\mathbb{Z} / m \mathbb{Z})^{\times}$ which corresponds to $\operatorname{Gal}(K / k)$ under the natural isomorphism

$$
(\mathbb{Z} / m \mathbb{Z})^{\times} \ni t \bmod m \mapsto(t, K) \in \operatorname{Gal}(K / \mathbb{Q}),
$$

where the automorphism $(t, K)$ maps $\zeta_{m}$ to $\zeta_{m}^{t}$. Throughout this paper, we use the following symbols:

- $q_{1}=p_{1}^{e_{1}}, q_{2}=p_{2}^{e_{2}}$;

- $\zeta=\zeta_{m}$

- $K_{1}=\mathbb{Q}\left(\zeta_{q_{1}}\right), K_{2}=\mathbb{Q}\left(\zeta_{q_{2}}\right)$

- $k_{1}=k \cap K_{1}, k_{2}=k \cap K_{2}$;

- $W$ is the group of roots of unity in $k$;

- $D\left(q_{1}\right)=\left\langle-1, \mathrm{~N}_{K_{1} / k_{1}}\left(1-\zeta^{a q_{2}}\right): 1 \leq a<q_{1} \wedge\left(a, p_{1}\right)=1\right\rangle$;

- $D\left(q_{2}\right)=\left\langle-1, \mathrm{~N}_{K_{2} / k_{2}}\left(1-\zeta^{q_{1} b}\right): 1 \leq b<q_{2} \wedge\left(b, p_{2}\right)=1\right\rangle$;

- $D(m)=\left\langle-1, \mathrm{~N}_{K / k}\left(1-\zeta^{x}\right): 1 \leq x<m \wedge\left(x, p_{1}\right)=\left(x, p_{2}\right)=1\right\rangle$;

- $D=D\left(q_{1}\right) D\left(q_{2}\right) D(m)$

- $C=D \cap E$ is the group of circular units in $k$;

- $T_{1}=\left\{a(=a \bmod m) \in T: a \equiv 1 \bmod q_{2}\right\}$;

- $T_{2}=\left\{b(=b \bmod m) \in T: b \equiv 1 \bmod q_{1}\right\}$;

- $T_{1}^{\prime}=\left\{a \in T_{1}\right.$ : there exists $b \in T_{2}$ such that $\left.a b \in N\right\}$;

- $T_{2}^{\prime}=\left\{b \in T_{2}\right.$ : there exists $a \in T_{1}$ such that $\left.a b \in N\right\}$; 
- $J=-1 \bmod m \in T$;

- $J_{1} \equiv-1 \bmod q_{1} \wedge J_{1} \equiv 1 \bmod q_{2}$;

- $J_{2} \equiv 1 \bmod q_{1} \wedge J_{2} \equiv-1 \bmod q_{2}$.

LEMMA 1. With the above notation we have:

(1) $\operatorname{Gal}\left(K_{1} / k_{1}\right)=T_{1}^{\prime}$,

(2) $\operatorname{Gal}\left(K_{2} / k_{2}\right)=T_{2}^{\prime}$,

(3) $\operatorname{Gal}\left(k / k_{1}\right)=T_{1}^{\prime} T_{2} / N$,

(4) $\operatorname{Gal}\left(k / k_{2}\right)=T_{1} T_{2}^{\prime} / N$.

Proof. Easy.

Remark. By the statements (1) and (2) of Lemma 1, we can see that $k_{i}$ is imaginary if and only if $J_{i} \notin T_{i}^{\prime}(i=1,2)$.

In order to prove the theorem, we have to consider the following four cases separately:

I. Both $k_{1}$ and $k_{2}$ are imaginary $\left(\Leftrightarrow J_{1} \notin T_{1}^{\prime} \wedge J_{2} \notin T_{2}^{\prime}\right)$;

II. $k_{1}$ is imaginary and $k_{2}$ is real $\left(\Leftrightarrow J_{1} \notin T_{1}^{\prime} \wedge J_{2} \in T_{2}^{\prime}\right)$;

II'. $k_{1}$ is real and $k_{2}$ is imaginary $\left(\Leftrightarrow J_{1} \in T_{1}^{\prime} \wedge J_{2} \notin T_{2}^{\prime}\right)$;

III. Both $k_{1}$ and $k_{2}$ are real $\left(\Leftrightarrow J_{1} \in T_{1}^{\prime} \wedge J_{2} \in T_{2}^{\prime}\right)$.

But case I was treated in [1], and cases II and $\mathrm{II}^{\prime}$ are similar. So it is sufficient to consider cases II and III. In case II, we use the following symbols:

- $a_{1}(=1), a_{2}, \ldots, a_{r_{1}}$ a system of representatives for $T_{1} /\left\langle J_{1}\right\rangle T_{1}^{\prime}$;

- $b_{1}(=1), b_{2}, \ldots, b_{r_{2}}$ a system of representatives for $T_{2} / T_{2}^{\prime}$;

- $d_{1}(=1), d_{2}, \ldots, d_{s}$ a system of representatives for $T_{1}^{\prime} T_{2}^{\prime} / N$;

- $Y=\left\{a_{i_{1}} b_{i_{2}} d_{j}: 1 \leq i_{1} \leq r_{1} \wedge 1 \leq i_{2} \leq r_{2} \wedge 1 \leq j \leq s\right\}$

- $Y^{\prime}=Y-\left\{1, a_{2}, \ldots, a_{r_{1}}, b_{2}, \ldots, b_{r_{2}}\right\}$;

- $M=\left\{a_{i_{1}} q_{2}: 2 \leq i_{1} \leq r_{1}\right\} \cup\left\{q_{1} b_{i_{2}}: 2 \leq i_{2} \leq r_{2}\right\} \cup Y^{\prime}$;

- $M^{\prime}=M \cup\{0\}$.

And, in case III, we use the following ones:

- $a_{1}(=1), a_{2}, \ldots, a_{r_{1}}$ a system of representatives for $T_{1} / T_{1}^{\prime}$;

- $b_{1}(=1), b_{2}, \ldots, b_{r_{2}}$ a system of representatives for $T_{2} / T_{2}^{\prime}$;

- $d_{1}(=1), d_{2}, \ldots, d_{s}$ a system of representatives for $T_{1}^{\prime} T_{2}^{\prime} /\langle J\rangle N$;

- $Y=\left\{a_{i_{1}} b_{i_{2}} d_{j}: 1 \leq i_{1} \leq r_{1} \wedge 1 \leq i_{2} \leq r_{2} \wedge 1 \leq j \leq s\right\}$;

- $Y^{\prime}=Y-\left\{1, a_{2}, \ldots, a_{r_{1}}, b_{2}, \ldots, b_{r_{2}}\right\}$

- $M=\left\{a_{i_{1}} q_{2}: 2 \leq i_{1} \leq r_{1}\right\} \cup\left\{q_{1} b_{i_{2}}: 2 \leq i_{2} \leq r_{2}\right\} \cup Y^{\prime}$;

- $M^{\prime}=M \cup\{0\}$.

R e mark. We note that $Y$ is a system of representatives for $T /\langle J\rangle N$. Further, the cardinality of $M$ is equal to $\frac{1}{2}[k: \mathbb{Q}]-1$, which is the unit rank of $k$. 
Now let $l: k^{\times} \rightarrow \mathbb{R}[G]$ be the $G$-module homomorphism defined by

$$
l: k^{\times} \ni \alpha \mapsto \sum_{\sigma \in G} \log \left|\alpha^{\sigma}\right| \cdot \sigma^{-1} \in \mathbb{R}[G] .
$$

It is easy to see that $\operatorname{Ker} l \cap E=\operatorname{Ker} l \cap C=W$. Hence

$$
[E: C]=[l(E): l(C)] \text {. }
$$

Furthermore, any finite subset $\left\{v_{1}, \ldots, v_{r}\right\}$ of $C$ is a system of fundamental circular units of $k$ if and only if $\left\{l\left(v_{1}\right), \ldots, l\left(v_{r}\right)\right\}$ is a $\mathbb{Z}$-basis of $l(C)$.

For any $a, b, y \in T$, we define circular units $v_{a q_{2}}, v_{q_{1} b}, v_{y}$ by

$v_{a q_{2}}=\mathrm{N}_{K_{1} / k_{1}}\left(\frac{1-\zeta^{a q_{2}}}{1-\zeta^{q_{2}}}\right), \quad v_{q_{1} b}=\mathrm{N}_{K_{2} / k_{2}}\left(\frac{1-\zeta^{q_{1} b}}{1-\zeta^{q_{1}}}\right), \quad v_{y}=\mathrm{N}_{K / k}\left(1-\zeta^{y}\right)$.

Then we notice the following facts:

(1) If $a \equiv a^{\prime} \bmod T_{2} N$, then $v_{a q_{2}}=v_{a^{\prime} q_{2}}$.

(2) If $b \equiv b^{\prime} \bmod T_{1} N$, then $v_{q_{1} b}=v_{q_{1} b^{\prime}}$.

(3) If $y \equiv y^{\prime} \bmod N$, then $v_{y}=v_{y^{\prime}}$.

Let $C^{\prime}$ be the subgroup of $C$ generated by $\left\{v_{x}: x \in M\right\}$. Later, we shall see that $l\left(C^{\prime}\right)$ has finite index in $l(C)$ and $l(E)$. Hence we have

$$
[E: C]=[l(E): l(C)]=\frac{\left[l(E): l\left(C^{\prime}\right)\right]}{\left[l(C): l\left(C^{\prime}\right)\right]} .
$$

2. Computation of $\left[l(C): l\left(C^{\prime}\right)\right]$. First, from the definition of $C^{\prime}$, we can easily see the following lemma.

Lemma 2. $l\left(C^{\prime}\right)$ is generated by $\left\{l\left(v_{x}\right): x \in M\right\}$.

We choose two integers $l_{1}$ and $l_{2}$ such that

$$
\begin{aligned}
l_{1} & \equiv 1 \bmod q_{1}, \quad p_{1} l_{1} \equiv 1 \bmod q_{2}, \\
p_{2} l_{2} & \equiv 1 \bmod q_{1}, \\
l_{2} & \equiv 1 \bmod q_{2} .
\end{aligned}
$$

Then $l_{1} \in T_{2}$ and $l_{2} \in T_{1}$. We define $f_{1}, g_{1}, f_{2}$ and $g_{2}$ by

$$
\begin{array}{ll}
f_{1}=\left[\left\langle l_{1}\right\rangle T_{2}^{\prime}: T_{2}^{\prime}\right], & g_{1}=\left[T_{2}:\left\langle l_{1}\right\rangle T_{2}^{\prime}\right], \\
f_{2}=\left[\left\langle l_{2}\right\rangle T_{1}^{\prime}: T_{1}^{\prime}\right], & g_{2}=\left[T_{1}:\left\langle l_{2}\right\rangle T_{1}^{\prime}\right] .
\end{array}
$$

Let $\left\{1, s_{2}, \ldots, s_{g_{1}}\right\}$ and $\left\{1, t_{2}, \ldots, t_{g_{2}}\right\}$ be systems of representatives for $T_{2} /\left\langle l_{1}\right\rangle T_{2}^{\prime}$ and $T_{1} /\left\langle l_{2}\right\rangle T_{1}^{\prime}$ respectively.

Proposition 3. In case II, $l(C)$ is generated by

$$
l\left(v_{a_{i_{1}} q_{2}}\right) \quad\left(2 \leq i_{1} \leq r_{1}\right), \quad l\left(v_{y}\right) \quad\left(y \in Y^{\prime}\right)
$$

and

$$
\frac{1}{2} l\left(v_{q_{1} l_{1}}\right), \ldots, \frac{1}{2} l\left(v_{q_{1} l_{1}^{f_{1}-1}}\right),
$$




$$
\begin{array}{r}
l\left(v_{q_{1} s}\right), \frac{1}{2}\left(l\left(v_{q_{1} s}\right)-l\left(v_{q_{1} s l_{1}}\right)\right), \ldots, \frac{1}{2}\left(l\left(v_{q_{1} s} l_{1}^{f_{1}-2}\right)-l\left(v_{q_{1} s l_{1}^{f_{1}-1}}\right)\right) \\
\left(s=s_{2}, \ldots, s_{g_{1}}\right) .
\end{array}
$$

Moreover, $\left[l(C): l\left(C^{\prime}\right)\right]=2^{r_{2}-g_{1}}$.

Proposition 4. In case III, $l(C)$ is generated by

$$
l\left(v_{y}\right) \quad\left(y \in Y^{\prime}\right)
$$

and

$$
\begin{gathered}
\frac{1}{2} l\left(v_{q_{1} l_{1}}\right), \ldots, \frac{1}{2} l\left(v_{q_{1} l_{1}^{f_{1}-1}}\right), \\
l\left(v_{q_{1} s}\right), \frac{1}{2}\left(l\left(v_{q_{1} s}\right)-l\left(v_{q_{1} s l_{1}}\right)\right), \ldots, \frac{1}{2}\left(l\left(v_{q_{1} s l_{1}^{f_{1}-2}}\right)-l\left(v_{q_{1} s l_{1}^{f_{1}-1}}\right)\right) \\
\quad\left(s=s_{2}, \ldots, s_{g_{1}}\right)
\end{gathered}
$$

and

$$
\begin{gathered}
\frac{1}{2} l\left(v_{l_{2} q_{2}}\right), \ldots, \frac{1}{2} l\left(v_{l_{2}^{f_{2}-1} q_{2}}\right), \\
l\left(v_{t q_{2}}\right), \frac{1}{2}\left(l\left(v_{t q_{2}}\right)-l\left(v_{t l_{2} q_{2}}\right)\right), \ldots, \frac{1}{2}\left(l\left(v_{t l_{2}^{f_{2}-2} q_{2}}\right)-l\left(v_{t l_{2}^{f_{2}-1} q_{2}}\right)\right) \\
\quad\left(t=t_{2}, \ldots, t_{g_{2}}\right) .
\end{gathered}
$$

Moreover, $\left[l(C): l\left(C^{\prime}\right)\right]=2^{r_{1}+r_{2}-g_{1}-g_{2}}$.

Remark. In the next section, we shall see that $l\left(C^{\prime}\right)$ has finite index in $l(E)$. Hence

$$
\operatorname{rank}_{\mathbb{Z}} l\left(C^{\prime}\right)=\operatorname{rank}_{\mathbb{Z}} l(C)=\operatorname{rank}_{\mathbb{Z}} l(E) .
$$

On the other hand, we notice that the cardinality of every system of generators for $l\left(C^{\prime}\right)$ or $l(C)$ stated in the above propositions is equal to the unit rank of $k$. This implies that these systems of generators are bases.

We can prove Propositions 3 and 4 in a similar fashion. So we only prove Proposition 3. Let $L$ be the subgroup of $\mathbb{R}[G]$ generated by the elements stated in the proposition.

First we prove $L \subset l(C)$. Fix a $b_{i_{2}}\left(1 \leq i_{2} \leq r_{2}\right)$. Then

$$
\begin{aligned}
& \sum_{\substack{1 \leq i_{1} \leq r_{1} \\
1 \leq j \leq s}} l\left(v_{a_{i_{1}} b_{i_{2}} d_{j}}\right) \\
& \quad=\sum_{\substack{1 \leq i_{1} \leq r_{1} \\
1 \leq j \leq s}} l\left(\mathrm{~N}_{K / k}\left(1-\zeta^{a_{i_{1}} b_{i_{2}} d_{j}}\right)\right) \\
& \quad=\frac{1}{2} \sum_{\substack{1 \leq i_{1} \leq r_{1} \\
1 \leq j \leq s}} l\left(\mathrm{~N}_{K / k}\left(1-\zeta^{a_{i_{1}} b_{i_{2}} d_{j}}\right)\right)+\frac{1}{2} \sum_{\substack{1 \leq i_{1} \leq r_{1} \\
1 \leq j \leq s}} l\left(\mathrm{~N}_{K / k}\left(1-\zeta^{J_{1} a_{i_{1}} b_{i_{2}} d_{j}}\right)\right)
\end{aligned}
$$




$$
\begin{aligned}
& =\frac{1}{2} \sum_{\substack{0 \leq h_{1} \leq 1 \\
1 \leq i_{1} \leq r_{1} \\
1 \leq j \leq s}} l\left(\mathrm{~N}_{K / k}\left(1-\zeta^{J_{1}^{h_{1}} a_{i_{1}} b_{i_{2}} d_{j}}\right)\right) \\
& =\frac{1}{2} l\left(\prod_{\substack{0 \leq h_{1} \leq 1 \\
1 \leq i_{1} \leq r_{1} \\
1 \leq j \leq s}} \mathrm{~N}_{K / k}\left(1-\zeta^{J_{1}^{h_{1}} a_{i_{1}} b_{i_{2}} d_{j}}\right)\right) .
\end{aligned}
$$

Now, by Lemma 1(4), we have

$$
\prod_{\substack{0 \leq h_{1} \leq 1 \\ 1 \leq i_{1} \leq r_{1} \\ 1 \leq j \leq s}} \mathrm{~N}_{K / k}\left(1-\zeta^{J_{1}^{h_{1}} a_{i_{1}} b_{i_{2}} d_{j}}\right)=\mathrm{N}_{k / k_{2}}\left(\mathrm{~N}_{K / k}\left(1-\zeta^{b_{i_{2}}}\right)\right) .
$$

So we get

$$
\begin{aligned}
\sum_{\substack{1 \leq i_{1} \leq r_{1} \\
1 \leq j \leq s}} l\left(v_{a_{i_{1}} b_{i_{2}} d_{j}}\right) & \\
= & \frac{1}{2} l\left(\mathrm{~N}_{k / k_{2}}\left(\mathrm{~N}_{K / k}\left(1-\zeta^{b_{i_{2}}}\right)\right)\right) \\
& =\frac{1}{2} l\left(\mathrm{~N}_{K_{2} / k_{2}}\left(\mathrm{~N}_{K / K_{2}}\left(1-\zeta^{b_{i_{2}}}\right)\right)\right) \\
& =\frac{1}{2} l\left(\mathrm{~N}_{K_{2} / k_{2}}\left(\frac{1-\zeta^{q_{1} b_{i_{2}}}}{1-\zeta^{q_{1} l_{1} b_{i_{2}}}}\right)\right) \\
& =\frac{1}{2} l\left(\mathrm{~N}_{K_{2} / k_{2}}\left(\frac{1-\zeta^{q_{1} b_{i_{2}}}}{1-\zeta^{q_{1}}}\right)\right)-\frac{1}{2} l\left(\mathrm{~N}_{K_{2} / k_{2}}\left(\frac{1-\zeta^{q_{1} l_{1} b_{i_{2}}}}{1-\zeta^{q_{1}}}\right)\right) \\
& =\frac{1}{2}\left(l\left(v_{q_{1} b_{i_{2}}}\right)-l\left(v_{q_{1} l_{1} b_{i_{2}}}\right)\right) .
\end{aligned}
$$

Hence

$$
\frac{1}{2}\left(l\left(v_{q_{1} b_{i_{2}}}\right)-l\left(v_{q_{1} l_{1} b_{i_{2}}}\right)\right) \in l(C) .
$$

From this, we can easily deduce that $L \subset l(C)$.

Next we prove $l(C) \subset L$. For this purpose, it is sufficient to prove that $l\left(v_{y}\right) \in L$ for $y \in Y-Y^{\prime}$, because it is obvious that $l\left(v_{q_{1} b_{i_{2}}}\right) \in L$ for $2 \leq i_{2} \leq r_{2}$. Fix an $a_{i_{1}}\left(2 \leq i_{1} \leq r_{1}\right)$. Then

$$
\begin{aligned}
\sum_{\substack{1 \leq i_{2} \leq r_{2} \\
1 \leq j \leq s}} l\left(v_{a_{i_{1}} b_{i_{2}} d_{j}}\right) & =l\left(\mathrm{~N}_{k / k_{1}}\left(\mathrm{~N}_{K / k}\left(1-\zeta^{a_{i_{1}}}\right)\right)\right) \\
& =l\left(\mathrm{~N}_{K_{1} / k_{1}}\left(\mathrm{~N}_{K / K_{1}}\left(1-\zeta^{a_{i_{1}}}\right)\right)\right) \\
& =l\left(\mathrm{~N}_{K_{1} / k_{1}}\left(\frac{1-\zeta^{a_{i_{1}} q_{2}}}{1-\zeta^{a_{i_{1}} q_{2} l_{2}}}\right)\right)
\end{aligned}
$$




$$
\begin{aligned}
& =l\left(\mathrm{~N}_{K_{1} / k_{1}}\left(\frac{1-\zeta^{a_{i_{1}} q_{2}}}{1-\zeta^{q_{2}}}\right)\right)-l\left(\mathrm{~N}_{K_{1} / k_{1}}\left(\frac{1-\zeta^{a_{i_{1}} q_{2} l_{2}}}{1-\zeta^{q_{2}}}\right)\right) \\
& =l\left(v_{a_{i_{1}} q_{2}}\right)-l\left(v_{a_{i_{1}} q_{2} l_{2}}\right) \in L .
\end{aligned}
$$

If $a_{i_{1}} b_{i_{2}} d_{j} \neq a_{i_{1}}$, then $a_{i_{1}} b_{i_{2}} d_{j} \in Y^{\prime}$ by the definition of $Y^{\prime}$. Hence $l\left(v_{a_{i_{1}}}\right) \in$ $L$. Now we fix a $b_{i_{2}}\left(1 \leq i_{2} \leq r_{2}\right)$. Then, as we have seen above,

$$
\sum_{\substack{1 \leq i_{1} \leq r_{1} \\ 1 \leq j \leq s}} l\left(v_{a_{i_{1}} b_{i_{2}} d_{j}}\right)=\frac{1}{2}\left(l\left(v_{q_{1} b_{i_{2}}}\right)-l\left(v_{q_{1} l_{1} b_{i_{2}}}\right)\right) .
$$

If $a_{i_{1}} b_{i_{2}} d_{j} \neq b_{i_{2}}$, then $l\left(v_{a_{i_{1}} b_{i_{2}} d_{j}}\right) \in L$. Hence $l\left(v_{b_{i_{2}}}\right) \in L$. We have thus proved that $l(C) \subset L$.

Finally, by computing the determinant of the transition matrix, we can see that

$$
\left[l(C): l\left(C^{\prime}\right)\right]=\frac{1}{\left(\frac{1}{2}\right)^{f_{1}-1} \cdot\left(\left(\frac{1}{2}\right)^{f_{1}-1}\right)^{g_{1}-1}}=2^{f_{1} g_{1}-g_{1}}=2^{r_{2}-g_{1}} .
$$

This completes the proof of Proposition 3.

3. Computation of $\left[l(E): l\left(C^{\prime}\right)\right]$. For each $t \in T$, we let

$$
\begin{aligned}
c_{0} & =1, \\
c_{t q_{2}} & = \begin{cases}1 & \text { if } t \in T_{2} N, \\
0 & \text { otherwise, }\end{cases} \\
c_{t q_{1}} & = \begin{cases}1 & \text { if } t \in T_{1} N, \\
0 & \text { otherwise, }\end{cases} \\
c_{t} & = \begin{cases}1 & \text { if } t \in N, \\
0 & \text { otherwise }\end{cases}
\end{aligned}
$$

Further, we define

$$
\begin{aligned}
b_{0, t}= & 2, \\
b_{a q_{2}, t}= & \left(c_{\left(a q_{2}\right) t}+c_{J\left(a q_{2}\right) t}\right)-\left(c_{t q_{2}}+c_{J t q_{2}}\right) \quad(a \in T), \\
b_{q_{1} b, t}= & \left(c_{\left(q_{1} b\right) t}+c_{J\left(q_{1} b\right) t}\right)-\left(c_{t q_{1}}+c_{J t q_{1}}\right) \quad(b \in T), \\
b_{y, t}= & \left(c_{y t}+c_{J y t}\right)-\frac{1}{\left[k: k_{2}\right]}\left(c_{y t l_{1} q_{1}}+c_{J y t l_{1} q_{1}}\right) \\
& -\frac{1}{\left[k: k_{1}\right]}\left(c_{y t l_{2} q_{2}}+c_{J y t l_{2} q_{2}}\right)+\frac{2}{[k: \mathbb{Q}]} \quad(y \in T) .
\end{aligned}
$$

We denote by $\sigma_{t}(t \in T)$ the automorphism of $k$ over $\mathbb{Q}$ which is the image of $(t, K)$ under the canonical surjection

$$
\operatorname{Gal}(K / \mathbb{Q}) \rightarrow G=\operatorname{Gal}(k / \mathbb{Q}) .
$$


We omit the proof of the next lemma because it is just the same as those of Lemmas 3.5 and 3.6 in [1].

Lemma 5. Let $Q$ be the unit index of $k$ and $h^{+}$the class number of $k^{+}$. Then

$$
\left[l(E): l\left(C^{\prime}\right)\right]=\frac{1}{[k: \mathbb{Q}]} \cdot\left|\operatorname{det}\left(b_{x, t}\right)_{x \in M^{\prime}, t \in Y}\right| \cdot Q h^{+} .
$$

In what follows, we compute the determinant of the matrix $\left(b_{x, t}\right)_{x \in M^{\prime}, t \in Y}$. We define

$$
A=\left\{J_{1}^{h_{1}} a_{i_{1}} q_{2}: 0 \leq h_{1} \leq 1 \wedge 1 \leq i_{1} \leq r_{1}\right\}, \quad B=\left\{q_{1} b_{i_{2}}: 1 \leq i_{2} \leq r_{2}\right\}
$$

in case II, and

$$
A=\left\{a_{i_{1}} q_{2}: 1 \leq i_{1} \leq r_{1}\right\}, \quad B=\left\{q_{1} b_{i_{2}}: 1 \leq i_{2} \leq r_{2}\right\}
$$

in case III. Then it is easy to see that

$$
\sum_{y \in A} c_{y t}=\sum_{y \in B} c_{y t}=1
$$

for any $t \in T$.

Lemma 6. (1) For any $x \in A$, there exist rational numbers $\alpha_{i_{1}}\left(1 \leq i_{1}\right.$ $\left.\leq r_{1}\right)$ such that

$$
c_{x t}+c_{J x t}=\alpha_{1}\left(c_{0}+c_{0}\right)+\sum_{i_{1}=2}^{r_{1}} \alpha_{i_{1}}\left(c_{\left(a_{i_{1}} q_{2}\right) t}+c_{J\left(a_{i_{1}} q_{2}\right) t}\right)
$$

for any $t \in T$.

(2) For any $x \in B$, there exist rational numbers $\beta_{i_{2}}\left(1 \leq i_{2} \leq r_{2}\right)$ such that

$$
c_{x t}+c_{J x t}=\beta_{1}\left(c_{0}+c_{0}\right)+\sum_{i_{2}=2}^{r_{2}} \beta_{i_{2}}\left(c_{\left(q_{1} b_{i_{2}}\right) t}+c_{J\left(q_{1} b_{i_{2}}\right) t}\right)
$$

for any $t \in T$.

Proof. As cases II and III are similar, we prove the lemma for case II. If $x \in M$, there is nothing to prove. So we suppose that $x \notin M$.

(1) Since

$$
c_{\left(J_{1} a_{i_{1}} q_{2}\right) t}+c_{J\left(J_{1} a_{i_{1}} q_{2}\right) t}=c_{\left(J_{1} a_{i_{1}} q_{2}\right) t}+c_{\left(a_{i_{1}} q_{2}\right) t}=c_{\left(a_{i_{1}} q_{2}\right) t}+c_{J\left(a_{i_{1}} q_{2}\right) t},
$$

it suffices to consider the case $x=q_{2}$. Let us define

$$
A_{0}=\left\{a_{i_{1}} q_{2}: 1 \leq i_{1} \leq r_{1}\right\}, \quad A_{1}=\left\{J_{1} a_{i_{1}} q_{2}: 1 \leq i_{1} \leq r_{1}\right\} .
$$

Then

$$
\sum_{y \in A_{0}}\left(c_{y t}+c_{J y t}\right)=\sum_{y \in A}\left(c_{y t}+c_{J y t}\right)-\sum_{y \in A_{1}}\left(c_{y t}+c_{J y t}\right) .
$$


On the other hand, we can see that

$$
\sum_{y \in A_{1}}\left(c_{y t}+c_{J y t}\right)=\sum_{y \in A_{1}}\left(c_{(J y) t}+c_{J(J y) t}\right)=\sum_{y \in A_{0}}\left(c_{y t}+c_{J y t}\right) .
$$

Hence

$$
\sum_{y \in A_{0}}\left(c_{y t}+c_{J y t}\right)=\frac{1}{2} \sum_{y \in A}\left(c_{y t}+c_{J y t}\right)=\frac{1}{2}(1+1)=\frac{1}{2}\left(c_{0}+c_{0}\right) .
$$

Consequently, we obtain

$$
c_{q_{2} t}+c_{J q_{2} t}=\frac{1}{2}\left(c_{0}+c_{0}\right)-\sum_{i_{1}=2}^{r_{1}}\left(c_{\left(a_{i_{1}} q_{2}\right) t}+c_{J\left(a_{i_{1}} q_{2}\right) t}\right) .
$$

(2) From $x \notin M$, we have $x=q_{1}$. Since

$$
\sum_{i_{2}=1}^{r_{2}}\left(c_{\left(q_{1} b_{i_{2}}\right) t}+c_{J\left(q_{1} b_{i_{2}}\right) t}\right)=1+1=c_{0}+c_{0}
$$

we get

$$
c_{q_{1} t}+c_{J q_{1} t}=\left(c_{0}+c_{0}\right)-\sum_{i_{2}=2}^{r_{2}}\left(c_{\left(q_{1} b_{i_{2}}\right) t}+c_{J\left(q_{1} b_{i_{2}}\right) t}\right) .
$$

Using the above lemma, we can deduce that

$$
\left|\operatorname{det}\left(b_{x, t}\right)_{x \in M^{\prime}, t \in Y}\right|=r_{1} r_{2} \cdot\left|\operatorname{det}\left(c_{x t}+c_{J x t}\right)_{x \in M^{\prime}, t \in Y}\right|
$$

by the same argument as in the proof of Lemma 3.8 in [1]. So, in order to accomplish our purpose, we have to compute the determinant of the matrix $\left(c_{x t}+c_{J x t}\right)_{x \in M^{\prime}, t \in Y}$.

For each $t \in Y$, there exists exactly one $u \in Y$ such that $t u \in\langle J\rangle N$, because $Y$ is a system of representatives for $T /\langle J\rangle N$. We denote this $u$ by $t^{\prime}$. Then the map $Y \ni t \mapsto t^{\prime} \in Y$ is bijective.

Lemma 7. Suppose $x \in M, t \in Y$ and $c_{x t^{\prime}}+c_{J x t^{\prime}} \neq 0$. Then:

(1) if $x \in Y^{\prime}$, then $t=x$;

(2) if $x=a_{i_{1}} q_{2}\left(2 \leq i_{1} \leq r_{1}\right)$, then $t \in Y^{\prime}$ or $t=a_{i_{1}}$;

(3) if $x=q_{1} b_{i_{2}}\left(2 \leq i_{2} \leq r_{2}\right)$, then $t \in Y^{\prime}$ or $t=b_{i_{2}}$.

P r o of. (1) Straightforward.

(2) If $c_{\left(a_{i_{1}} q_{2}\right) t^{\prime}}+c_{J\left(a_{i_{1}} q_{2}\right) t^{\prime}} \neq 0$, then $a_{i_{1}} t^{\prime} \in\langle J\rangle T_{2} N$. Hence there is a $y=b_{i_{2}} J^{h} d_{j}$ such that $a_{i_{1}} y t^{\prime}=a_{i_{1}} b_{i_{2}} J^{h} d_{j} t^{\prime} \in\langle J\rangle N$, and so we have $a_{i_{1}} b_{i_{2}} d_{j} t^{\prime} \in\langle J\rangle N$. Therefore $t=a_{i_{1}} b_{i_{2}} d_{j}$, and we obtain $t=a_{i_{1}}$ or $t \in Y^{\prime}$.

(3) In case III, the proof is similar to (2). So we consider case II. If $c_{\left(q_{1} b_{i_{2}}\right) t^{\prime}}+c_{J\left(q_{1} b_{i_{2}}\right) t^{\prime}} \neq 0$, then $b_{i_{2}} t^{\prime} \in\langle J\rangle T_{1} N$. Hence there is a $w=J_{1}^{h_{1}} a_{i_{1}} d_{j}$ such that $w b_{i_{2}} t^{\prime}=J_{1}^{h_{1}} a_{i_{1}} b_{i_{2}} d_{j} t^{\prime} \in\langle J\rangle N$, and so $a_{i_{1}} b_{i_{2}} J_{2}^{h_{1}} d_{j} t^{\prime} \in\langle J\rangle N$. As 
$J_{2} \in T_{2}^{\prime}$, there exists a $d_{j^{\prime}}$ such that $J_{2}^{h_{1}} d_{j} \equiv d_{j^{\prime}} \bmod \langle J\rangle N$, and then $a_{i_{1}} b_{i_{2}} d_{j^{\prime}} t^{\prime} \in\langle J\rangle N$. Therefore $t=b_{i_{2}}$ or $t \in Y^{\prime}$.

From this, we can obtain the following conclusion.

Proposition 8. Let $Q$ be the unit index of $k$ and $h^{+}$the class number of $k^{+}$.

(1) In case II, we have

$$
\left[l(E): l\left(C^{\prime}\right)\right]=\frac{\left[k_{1}: \mathbb{Q}\right]\left[k_{2}: \mathbb{Q}\right]}{[k: \mathbb{Q}]} \cdot 2^{r_{2}-1} \cdot Q h^{+} .
$$

(2) In case III, we have

$$
\left[l(E): l\left(C^{\prime}\right)\right]=\frac{\left[k_{1}: \mathbb{Q}\right]\left[k_{2}: \mathbb{Q}\right]}{[k: \mathbb{Q}]} \cdot 2^{r_{1}+r_{2}-1} \cdot Q h^{+} .
$$

In particular, $l\left(C^{\prime}\right)$ and $l(C)$ have finite indices in $l(E)$.

4. Proof of Theorem B. First we consider case II. By Propositions 3 and $8(1)$, we have

$$
\begin{aligned}
{[E: C] } & =\frac{\left[l(E): l\left(C^{\prime}\right)\right]}{\left[l(C): l\left(C^{\prime}\right)\right]}=\frac{\left[k_{1}: \mathbb{Q}\right]\left[k_{2}: \mathbb{Q}\right]}{[k: \mathbb{Q}]} \cdot \frac{2^{r_{2}-1}}{2^{r_{2}-g_{1}}} \cdot Q h^{+} \\
& =\frac{\left[k_{1}: \mathbb{Q}\right]\left[k_{2}: \mathbb{Q}\right]}{[k: \mathbb{Q}]} \cdot 2^{g_{1}-1} \cdot Q h^{+} .
\end{aligned}
$$

Now, since $\sigma_{l_{1}}$ is the inverse of the Frobenius automorphism for $p_{1}$ in $k$, we have

Therefore

$$
g_{1}=\left[T:\left\langle l_{1}\right\rangle T_{1} N\right]=\left[G: D_{p_{1}}\right] .
$$

$$
[E: C]=\frac{\left[k_{1}: \mathbb{Q}\right]\left[k_{2}: \mathbb{Q}\right]}{[k: \mathbb{Q}]} \cdot 2^{\left[G: D_{p_{1}}\right]-1} \cdot Q h^{+} .
$$

Next we consider case III. By Propositions 4 and 8(2), we have

$$
[E: C]=\frac{\left[k_{1}: \mathbb{Q}\right]\left[k_{2}: \mathbb{Q}\right]}{[k: \mathbb{Q}]} \cdot 2^{g_{1}+g_{2}-1} \cdot Q h^{+} .
$$

As also $g_{1}=\left[G: D_{p_{1}}\right]$ and $g_{2}=\left[G: D_{p_{2}}\right]$, we obtain

$$
[E: C]=\frac{\left[k_{1}: \mathbb{Q}\right]\left[k_{2}: \mathbb{Q}\right]}{[k: \mathbb{Q}]} \cdot 2^{\left[G: D_{p_{1}}\right]+\left[G: D_{p_{2}}\right]-1} \cdot Q h^{+} .
$$

This completes the proof of the theorem.

5. The real case. Let $k$ be a real abelian number field with conductor $p_{1}^{e_{1}} p_{2}^{e_{2}}$. Let $T_{1}, T_{2}, T_{1}^{\prime}, T_{2}^{\prime}, N$ be the same as in Section 1 , and 
$\left\{a_{i_{1}}\right\},\left\{b_{i_{2}}\right\},\left\{d_{j}\right\}$ be systems of representatives for $T_{1} / T_{1}^{\prime}, T_{2} / T_{2}^{\prime}, T_{1}^{\prime} T_{2}^{\prime} / N$ respectively. Then

$$
\left\{v_{a_{i_{1}} q_{2}}: 2 \leq i_{1} \leq r_{1}\right\} \cup\left\{v_{q_{1} b_{i_{2}}}: 2 \leq i_{2} \leq r_{2}\right\} \cup\left\{v_{y}: y \in Y^{\prime}\right\}
$$

is a system of fundamental circular units of $k$, where

$$
\begin{aligned}
Y^{\prime}= & \left\{a_{i_{1}} b_{i_{2}} d_{j}: 1 \leq i_{1} \leq r_{1} \wedge 1 \leq i_{2} \leq r_{2} \wedge 1 \leq j \leq s\right\} \\
& -\left\{1, a_{2}, \ldots, a_{r_{1}}, b_{2}, \ldots, b_{r_{2}}\right\} .
\end{aligned}
$$

We can deduce Sinnott's index formula

$$
[E: C]=\frac{\left[k_{1}: \mathbb{Q}\right]\left[k_{2}: \mathbb{Q}\right]}{[k: \mathbb{Q}]} \cdot 2^{[k: \mathbb{Q}]-1} \cdot h
$$

by computing the regulator of the system of fundamental units. We omit the proof because it is very similar to that of the imaginary case.

Acknowledgments. I am grateful to Radan Kučera for many helpful comments and suggestions for improving an earlier version of this note. I would like to thank the referee for his careful reading of the manuscript and valuable suggestions.

\section{References}

[1] K. Dohmae, On bases of groups of circular units of some imaginary abelian number fields, J. Number Theory 61 (1996), 343-364.

[2] R. Gold and J. Kim, Bases for cyclotomic units, Compositio Math. 71 (1989), $13-28$.

[3] R. Kučera, On bases of odd and even universal ordinary distributions, J. Number Theory 40 (1992), 264-283.

[4] - On bases of the Stickelberger ideal and of the group of circular units of a cyclotomic field, ibid., 284-316.

[5] W. Sinnott, On the Stickelberger ideal and the circular units of a cyclotomic field, Ann. of Math. 108 (1978), 107-134.

[6] -, On the Stickelberger ideal and the circular units of an abelian field, Invent. Math. 62 (1980), 181-234.

[7] L. Washington, Introduction to Cyclotomic Fields, Grad. Texts in Math. 83, Springer, New York, 1980.

Department of Mathematics

Tokyo Metropolitan University

Minami-Ohsawa 1-1, Hachioji-shi

Tokyo 192-03, Japan

E-mail: dohmae@math.metro-u.ac.jp

Received on 26.9.1996

and in revised form on 7.4 .1997 